\title{
Percepcions dels estudiants sobre el valor de les tecnologies de la informació i comunicació en la seva formació com a docents
}

\section{Ricardo Domínguez *}

\section{Resum}

Les actituds i creences dels docents al voltant de les tecnologies exerceixen un factor clau per a la implementació de les mateixes en la pràctica educativa. Els programes de formació del professorat en competència TIC, tant en la formació inicial com en la permanent, solen centrar-se en l'adquisició d'una sèrie de coneixements i en el desenvolupament de certes habilitats i destreses entorn de l'ús dels recursos tecnològics, però no solen abordar les percepcions o creences que tenen els docents sobre el seu valor en els processos d'ensenyament-aprenentatge. En aquest article es presenta una recerca, realitzada en el context de la formació inicial de futurs docents de Primària, en la qual s'han generat espais d'autoreflexió sobre el valor i ús dels recursos tecnològics. A través del qüestionari realitzat a final del curs s'ha analitzat quins aspectes són destacats pels estudiants sobre l'ús de les tecnologies en la seva formació i en la seva utilització a l'aula.

\section{Paraules clau}

Percepcions, creences, actituds, tecnologies de la informació i comunicació, formació inicial, gènere.

Recepció original: 28 de maig de 2019

Acceptació: 06 de març de 2020

Publicació: 30 de juny de 2020

\section{Introducció}

Existeix un debat significatiu sobre els factors que poden afavorir una integració efectiva de les noves tecnologies digitals en la pràctica educativa dels docents. Aquest debat en diversos estudis s'ha traslladat a l'anàlisi d'aquells factors que poden dificultar aquest procés d'adaptació, categoritzant-los com a barreres de primer i segon ordre (Brickner, 1995; Ertmer, 1999; Pelgrum, 2001).

Les barreres de primer ordre són definides com a extrínseques als docents referintse, entre altres qüestions, a la infraestructura, suport tècnic i estructura organitzativa dels centres, i a la capacitació o competència digital del professorat. Aquestes barreres són fàcilment mesurables, per la qual cosa els primers passos s'han orientat generalment a incrementar la dotació tecnològica de les institucions educatives i a implementar en els programes de formació del professorat, tant en la formació inicial com permanent, aspectes que abordin la competència digital. No obstant això, aquests esforços a vegades no s'han traduït en una implementació posterior en la pràctica educativa.

En aquest sentit es refereix també l'existència de barreres de segon ordre o intrínseques al docent, constituïdes fonamentalment per les seves creences i actituds, que exerceixen un paper clau per a explicar la pràctica a l'aula i l'adopció de nous recursos i metodologies (Kagan, 1992; Pajares, 1992). En la construcció de les creences intervenen factors

(*) Professor del Departament de Didàctica de l'Expressió Musical, Plàstica i Corporal de la Facultat de Magisteri de la Universitat de València. Membre de CREARI (Grup de Recerca en Pedagogies Culturals). Adreça electrónica: ricardo.dominguez@uv.es 
subjectius i biogràfics en els quals tenen un gran pes els models observats a través dels anys com a estudiants i les primeres experiències com a docents (Nespor, 1987), convertint-se en un marc que es construeix gradualment, actuant de guia en les decisions a l'aula.

La majoria de les recerques afirmen que existeix una correspondència entre les creences sobre l'ús de les tecnologies i la manera en què aquestes són implementades en els processos d'ensenyament-aprenentatge (Ertmer, 2005; Lawless i Pellegrino, 2007), i del caràcter bidireccional entre creences i pràctica educativa. D'aquesta manera les actituds o percepcions positives cap a les tecnologies i sobre la pròpia autoeficàcia o competència digital estableixen condicions favorables per al seu ús a l'aula (Paraskeva, Bouta i Papagianni, 2008), mentre que una percepció negativa o una experiència traumàtica en el seu ús poden actuar com a inhibidors de la seva utilització.

D'aquestes consideracions deriva la necessitat d'incorporar en la formació del professorat, juntament amb una adequada capacitació en la competència digital, l'observació d'experiències d'èxit en relació amb l'ús de les tecnologies a l'aula, que augmentin la percepció que la seva adopció és viable, i l'habilitació d'espais de reflexió que permetin fer explícites les creences dels docents entorn del seu ús educatiu.

Diversos estudis han indagat en les percepcions i actituds cap a les TIC dels estudiants en la seva formació inicial com a futurs mestres. L'estudi realitzat per Gutíerrez, Palacios i Torrego (2010) indica el paper rellevant de la formació inicial en la construcció d'actituds positives cap a la integració curricular de les TIC. Destaquem també la recerca realitzada per Roig-Vila, Mengual-Andrés, Sterrantino i Quinto (2015), en la seva anàlisi de les actituds tenint en compte factors com l'edat, el gènere o la titulació de procedència. $O$ en el context de la formació inicial de docents de Secundària l'estudi dut a terme per Huerta i Domínguez (2011).

\section{Metodologia de l'estudi}

A partir d'aquestes qüestions, i del paper rellevant de les creences sobre l'ús de la tecnologia en els contextos educatius, es planteja la present recerca, en la qual analitzem el valor que té per a l'alumnat la utilització dels recursos tecnològics en la seva formació inicial com a futurs docents.

Per a dur a terme aquesta recerca es va optar per una metodologia mixta o plural (Alonso-Sanz, 2013; Martínez, Dimitriadis, Gómez, Rubia i De la Fuente, 2003), amb un enfocament predominant quantitatiu, basada en un estudi de cas amb una finalitat descriptiva (Yin, 2009).

\section{Objectius}

Com a objectius de la recerca s'estableixen:

1. Analitzar les actituds i valor atorgat pels estudiants de nivell de Grau de Magisteri respecte a les TIC en la seva formació.

2. Identificar si existeixen diferències significatives en relació amb el valor atorgat a les eines TIC en funció de gènere.

3. Introduir elements de reflexió sobre el valor de les TIC en la formació. 


\section{Estratègies metodològiques}

Per a recaptar les dades de l'estudi es va adaptar el qüestionari utilitzat per Domínguez (2019), en la seva recerca sobre l'anàlisi dels entorns personals d'aprenentatge en la formació de docents, ajustant el nombre d'ítems. Les dades posteriorment van ser analitzatdes a través del paquet informàtic SPSS v15.0 per a Windows. El qüestionari va ser implementat al final del curs, sent l'alumnat prèviament informat sobre l'objectiu d'aquest, del seu caràcter anònim i voluntari, i de la seva nul-la repercussió en la qualificació de l'assignatura. L'estructura del mateix es va organitzar en tres apartats:

- Dades socio-acadèmiques. Inclou dos ítems (edat i gènere).

- Valoració sobre la importància de les eines tecnològiques en la formació docent. Consta de 25 ítems tipus Likert amb una gradació en les alternatives de resposta de cinc nivells (Totalment en desacord, En desacord, Indiferent, D'acord, Totalment d'acord). Amb la finalitat de poder realitzar els estadístics descriptius de cada variable, les respostes s'han codificat numèricament. Els ítems favorables han estat codificats tal com podem observar en la taula 1 . Els ítems desfavorables $(6,12$ i 25$)$ se'ls ha assignat una puntuació inversa.

Taula 1. Codificació numèrica de les respostes dels ítems favorables

\begin{tabular}{lc}
\hline \multicolumn{1}{c}{ Respostes } & Codificació numèrica \\
\hline Totalment en desacord & 1 \\
En desacord & 2 \\
Indiferent & 3 \\
D'acord & 4 \\
Totalment d'acord & 5 \\
\hline
\end{tabular}

- Reflexió final. Inclou dues preguntes de resposta oberta. En la primera d'elles els subjectes han de realitzar una reflexió sobre si l'ús de les eines tecnològiques ha introduït canvis en la seva manera d'aprendre. En l'última qüestió els subjectes poden introduir qualsevol reflexió o comentari final.

La consistència interna del qüestionari va ser contrastada a través de la prova alfa de Cronbach, que mostra una fiabilitat acceptable per al conjunt dels ítems $(a=.79)(\mathrm{Kem}-$ ber, Biggs i Leung, 2004).

\section{Context i participants de l'estudi}

La població a la qual es refereix aquest estudi està constituïda per l'alumnat que cursa l'assignatura Programari i Maquinari en Contextos Educatius durant el curs 2017-2018 a la Universitat de València. Aquesta assignatura optativa de 6 crèdits es cursa durant el segon quadrimestre del curs i s'emmarca en els estudis de Grau en Mestre/a en Educació Primària de la Universitat de València.

La selecció de la mostra es va realitzar a través d'un mostreig no probabilístic de caràcter incidental o per conveniència (Blaxter, Hughes i Tight, 2008), al ser el propi investigador docent del grup. 
Com a suport a les classes presencials el grup va disposar d'una plataforma virtual Moodle, a través de la qual es van compartir recursos, es van enviar les tasques i es va distribuir el qüestionari utilitzat en la recerca.

Els continguts del curs es van organitzar en quatre blocs:

- Entorns personals d'aprenentatge (Personal Learning Environment).

- Maquinari i programari per a la creació i edició de continguts digitals.

- Videojocs i educació.

- Internet com a biblioteca de recursos i com a mitjà d'aprenentatge.

Al llarg del curs els participants van desenvolupar un portafoli digital fent ús d'un bloc individual. Com a eina per a la seva creació es va optar per la plataforma gratuïta Blogger', donada la seva facilitat d'ús.

Ens plantegem a partir del mateix que l'alumnat desenvolupara habilitats d'autoreflexió sobre el seu aprenentatge i mostrara evidències d'acompliment d'aquest, assumint un paper actiu en el procés d'ensenyament-aprenentatge (Barragán, 2005).

(...) los propósitos que guían el portafolios hacen referencia a la potencialidad de la herramienta para evidenciar cómo se van produciendo los procesos de enseñanza y aprendizaje desde dentro, es decir, desde el punto de vista -la voz- de los protagonistas. De este modo, es el propio sujeto el que organiza su trayectoria de reflexión en diferentes momentos a lo largo del proceso e 'inventa' su propio camino; el portafolios es, en definitiva, un recurso para la vida que pone de manifiesto las señas de identidad de sus protagonistas. (Agra, Gewerc i Montero, 2003, p. 104)

El portafoli digital es va estructurar en dues pàgines. En la pàgina principal els estudiants van dur a terme un diari d'aula, publicant una entrada al final de cada sessió en la qual compartien una reflexió sobre les tasques realitzades i continguts tractats. La pàgina secundària del portafoli va ser utilitzada per a compartir evidències d'acompliment mitjançant enllaços a les activitats realitzades en l'assignatura.

En el portafoli, cada estudiant va personalitzar la capçalera, va incloure una llicència Creative Commons ${ }^{2}$, el núvol o llista d'etiquetes utilitzades en les entrades publicades, i va agregar un gadget amb el llistat de portafolis de la resta de participants (figura 1).

\footnotetext{
(1) https://www.blogger.com

(2) https://creativecommons.org
} 
Figura 1. Portafoli digital [Captura de pantalla]

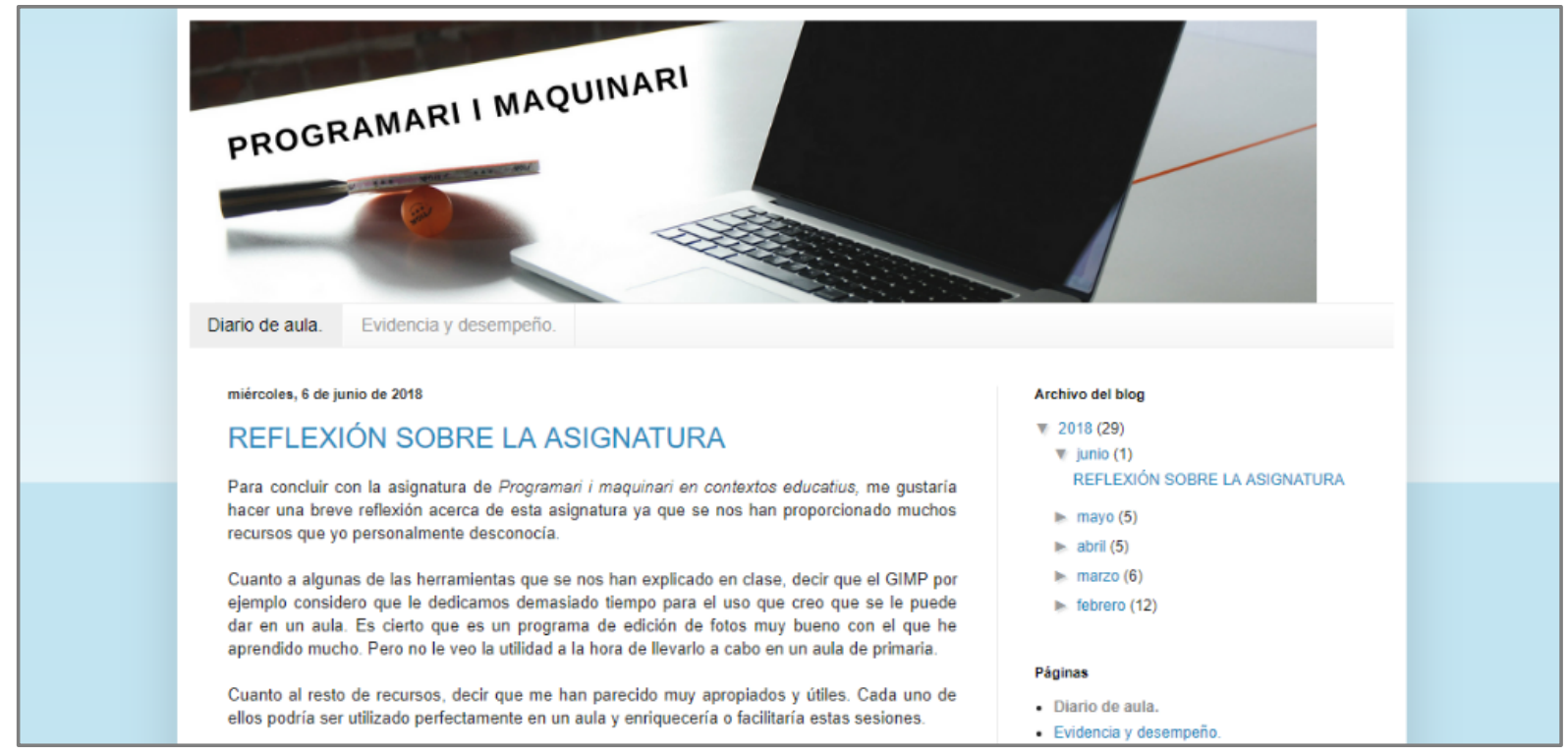

Font: https://programarimaquinaridisa.blogspot.com/

\section{Resultats}

En el qüestionari, voluntari i realitzat de manera anònima, participa el $87.2 \%$ del grup.

La mostra està composta per 41 participants. El 53.7\% dones $(n=22)$ i el $46.3 \%$ homes $(n=19)$. Per edats el $48.8 \%(n=20)$ té 20 o 21 anys, el $36.6 \%(n=15)$ té 22 o 23 , el 12.2\% $(n=5)$ té 24 o 25 anys, i el $2.4 \%(n=1) 26$ anys o més.

En una primera anàlisi descriptiva s'analitzen les mitjanes i desviacions típiques dels ítems 3-27, la taula 2 mostra un resum d'aquestes dades. Els resultats globals mostren que els participants realitzen una valoració positiva de l'ús dels recursos tecnològics en la seva formació $(M=4.03, D T=.282)$.

\section{Taula 2. Estadístics descriptius [v.3-v.27]}

\begin{tabular}{|c|c|c|}
\hline & M & $D T$ \\
\hline [v.3] Em permet accedir d'una manera més ràpida a la informació & 4.39 & .542 \\
\hline [v.4] És una ajuda per a gestionar de forma adequada la informació & 4.20 & .511 \\
\hline [v.5] És fonamental per a accedir a la informació actual & 4.29 & .750 \\
\hline [v.6] Suposa una càrrega de treball addicional & 2.49 & 1.143 \\
\hline $\begin{array}{l}\text { [v.7] Ajuda a desenvolupar una capacitat crítica cap a les tecnologies de la informació i comu- } \\
\text { nicació }\end{array}$ & 4.12 & .510 \\
\hline [v.8] És important per a contrastar la fiabilitat de la informació & 4.20 & 601 \\
\hline [v.9] Afavoreix l'adquisició de coneixements & 4.17 & .543 \\
\hline [v.10] Permeten desenvolupar les estratègies de cerca i selecció de la informació & 4.34 & .530 \\
\hline [v.11] Afavoreixen la re-descripció d'idees i el pensament creatiu & 4.05 & .705 \\
\hline [v.12] La diversitat de serveis en la xarxa dificulta la gestió dels seus continguts & 2.29 & .844 \\
\hline [v.13] Afavoreixen l'autonomia en els processos formatius & 4.05 & .631 \\
\hline
\end{tabular}


[v.14] És fonamental en la formació permanent del docent

[v.15] És una important ajuda en els processos de recerca en educació

[v.16] Afavoreix la capacitat per a adaptar la meva formació a noves situacions

[v.17] Afavoreix la generació de pensament propi

[v.18] Facilita l'intercanvi d'informació a través de diferents mitjans

[v.19] Ajuda a fomentar les habilitats comunicatives

[v.20] Faciliten el treball col-laboratiu

[v.21] Les meves aportacions en la xarxa poden afavorir l'aprenentatge d'altres persones

[v.22] Promouen la interactivitat en l'aprenentatge, el treball en grup i en equip

[v.23] Les contribucions d'altres usuaris en la xarxa afavoreixen el meu aprenentatge

[v.24] L'exposició pública dels processos d'aprenentatge afavoreix la reflexió personal

[v.25] L'exposició pública dels processos d'aprenentatge limita la llibertat d'expressió

[v.26] Afavoreix la construcció de la identitat docent, mitjançant la participació en xarxes professionals

[v.27] Són un important mitjà per a conèixer persones amb els mateixos interessos professionals

[GLOBAL]

A partir de les mitjanes estadístiques de cadascun dels ítems, observem com els aspectes millor valorats són que facilita l'intercanvi d'informació a través de diferents mitjans [v.18] $(M=4.51, D T=.597]$, resulten fonamentals en la formació permanent del docent [v.14] $(M=4.44, D T=.776)$, i que les aportacions personals en la xarxa poden afavorir l'aprenentatge d'altres persones [v.21] $(M=4.41, D T=.591)$.

En canvi, els aspectes pitjor valorats de l'ús de les TIC són que la diversitat de serveis en la xarxa dificulta la gestió dels seus continguts [v.12] $(M=2.29, D T=.844)$, suposen una càrrega de treball addicional [v.6] $(M=2.49, D T=1.143)$, i que l'exposició pública dels processos d'aprenentatge limita la llibertat d'expressió [v.25] ( $M=3.07, D T=.985)$.

A continuació, hem analitzat si existeixen diferències estadísticament significatives en els resultats en funció del gènere. Per a determinar el tipus de prova pertinent en l'anàlisi, prèviament s'ha comprovat la distribució normal de la mostra, a través de l'estadístic de Shapiro-Wilk (adequat per a mostres petites, inferiors a 50 subjectes); i l'homogeneïtat de la variància (homocedasticitat) per mitjà de l'estadístic de Levene.

En l'anàlisi de la variable [GLOBAL], obtinguda a partir de la mitjana de les variables 3-27 del qüestionari, s'ha fet ús de l'estadístic t de Student, en complir-se els supòsits de distribució normal, així com d'homogeneïtat de les variàncies, ja que la $p$ associada a les proves se situa en tots els casos per damunt del nivell de significació prefixat (.05) (vegeu taules 3 i 4). 
Taula 3. Proves de normalitat

\begin{tabular}{cccc}
\hline & Gènere & \multicolumn{2}{c}{ Shapiro-Wilk } \\
\hline & & Estadístic & p. \\
\hline \multirow{2}{*}{ GLOBAL] } & Home & .978 & .912 \\
& Dona & .937 & .169 \\
\hline
\end{tabular}

Taula 4. Prova d'homogeneïtat de la variància

\begin{tabular}{rrr}
\hline & Estadístic de Levene & $p$ \\
\hline [GLOBAL] & 1.487 & .230 \\
\hline
\end{tabular}

Tal com podem observar en la taula 5 , la valoració de l'ús dels recursos tecnològics en la formació és més positiva en les dones $(M=4.06, D T=.326)$ que en els homes $(M=$ $3.99, D T=.221)$, però l'estadístic $t$ ens revela que aquestes diferències no són estadísticament significatives $(p \geq .05)$.

Taula 5. Anàlisi comparativa en funció del gènere

\begin{tabular}{c|cc|cc|cc}
\hline & \multicolumn{2}{|c|}{ Home } & \multicolumn{2}{c|}{ Dona } & \multicolumn{2}{c}{ Prova $t$} \\
\hline & $\mathrm{M}$ & $\mathrm{DT}$ & $\mathrm{M}$ & $\mathrm{DT}$ & $\mathrm{t}$ & $\mathrm{p}$ \\
[GLOBAL] & 3.99 & .221 & 4.06 & .326 & -.865 & .392 \\
\hline
\end{tabular}

En una anàlisi més detallada hem observat si existeixen diferències significatives en funció del gènere en cadascun dels ítems del qüestionari. En aquest cas s'ha fet ús d'un estadístic no paramètric, la prova $U$ de Mann-Whitney, ja que després d'una primera anàlisi realitzatda amb la prova de Shapiro-Wilk, les dades obtingudes per a cadascun dels ítems no compleixen el supòsit de normalitat.

A partir de les dades obtingudes amb l'estadístic $U$, es pot observar que no existeixen diferències estadísticament significatives en cap dels ítems, ja que el valor $p$ és superior en tots els casos a .05. (vegeu taula 6).

Taula 6. Anàlisi comparativa en funció del gènere

\begin{tabular}{l|cc|cc|lc}
\hline Ítem & \multicolumn{2}{|c|}{ Home } & \multicolumn{2}{c|}{ Dona } & \multicolumn{2}{c}{ Prova U de Mann-Whitney } \\
\hline & $n_{1}$ & Rang mitjana & $n_{2}$ & Rang mitjana & $U$ & $p$ \\
\hline [v.3] & 19 & 20.37 & 22 & 21.55 & 197.000 & .718 \\
[v.4] & 19 & 19.05 & 22 & 22.68 & 172.000 & .224 \\
[v.5] & 19 & 21.74 & 22 & 20.36 & 195.000 & .688 \\
[v.6] & 19 & 18.92 & 22 & 22.80 & 169.500 & .287 \\
[v.7] & 19 & 20.63 & 22 & 21.32 & 202.000 & .813 \\
[v.8] & 19 & 17.63 & 22 & 23.91 & 145.000 & .053 \\
[v.9] & 19 & 17.87 & 22 & 23.70 & 149.500 & .057 \\
[v.10] & 19 & 19.26 & 22 & 22.50 & 176.000 & .311 \\
[v.11] & 19 & 20.21 & 22 & 21.68 & 194.000 & .668 \\
[v.12] & 19 & 20.39 & 22 & 21.52 & 197.500 & .748 \\
[v.13] & 19 & 18.76 & 22 & 22.93 & 166.500 & .176 \\
[v.14] & 19 & 20.95 & 22 & 21.05 & 208.000 & .976 \\
[v.15] & 19 & 22.37 & 22 & 19.82 & 183.000 & .448 \\
[v.16] & 19 & 18.39 & 22 & 23.25 & 159.500 & .147 \\
[v.17] & 19 & 22.13 & 22 & 20.02 & 187.500 & .536 \\
[v.18] & 19 & 20.76 & 22 & 21.20 & 204.500 & .893
\end{tabular}




\begin{tabular}{l|ll|ll|ll} 
[v.19] & 19 & 19.16 & 22 & 22.59 & 174.000 & .313 \\
[v.20] & 19 & 20.24 & 22 & 21.66 & 194.500 & .679 \\
[v.21] & 19 & 20.13 & 22 & 21.75 & 192.500 & .626 \\
[v.22] & 19 & 21.79 & 22 & 20.32 & 194.000 & .653 \\
[v.23] & 19 & 21.18 & 22 & 20.84 & 205.500 & .919 \\
[v.24] & 19 & 21.11 & 22 & 20.91 & 207.000 & .944 \\
[v.25] & 19 & 20.39 & 22 & 21.52 & 197.500 & .753 \\
[v.26] & 19 & 21.53 & 22 & 20.55 & 199.000 & .750 \\
[v.27] & 19 & 21.63 & 22 & 20.45 & 197.000 & .700 \\
\hline
\end{tabular}

La qüestió 28 , de tipus obert, ens permet recollir reflexions dels participants sobre la repercussió que tenen les tecnologies en el seu aprenentatge. La majoria de les respostes indiquen que les tecnologies estan produint un canvi significatiu en aquest. S'assenyala fonamentalment el seu impacte en les estratègies de cerca i selecció de la informació:

La manera de buscar informació ha canviat molt. En primer lloc dona moltíssima accessibilitat a una gran quantitat de continguts, la qual cosa és útil per a la recerca d'informació però al mateix temps hem d'aprendre a gestionar aquesta sobrequantitat d'informació.

Yo creo que el uso de estas herramientas sí ha cambiado mi manera de aprender ya que antes utilizaba mucho más el libro y ahora con todo lo que tenemos a nuestro alcance prácticamente ya no lo utilizo, pues para estudiar, realizar un trabajo, buscar información o cualquier otra cosa relacionada con el aprendizaje utilizo el ordenador, el móvil o cualquier dispositivo conectado a Internet.

Ha modificado mi manera de aprender debido a que he aprendido mediante otras herramientas totalmente distintas a las que anteriormente había conocido en otras etapas de mi vida acadèmica.

Sí, creo que es una manera óptima de buscar información, creo que las tecnologías aportan mucho a la educación y debemos de saber usarlas de manera crítica, buscar la información adecuada al contexto.

Entre les característiques més destacades s'indica la facilitat i rapidesa per a accedir a la informació:

Considere que les ferramentes tecnològiques són un avantatge. Recorde fa anys, quan els treballs de classe ho fèiem a mà o buscàvem el significat d'una paraula en el diccionari. Actualment, gràcies a les noves tecnologies crec que ens ha facilitat la rapidesa per a la recerca d'informació per a aprendre.

\section{També s'assenyala la seva contribució a l'aprenentatge autònom i col·laboratiu:}

Ha modificat la meua manera d'aprendre perquè ara sóc més autònoma. També m'ha facilitat crear materials segons necessitats i interessos, i conèixer gran quantitat de ferramentes útils per al treball docent.

Creo que los recursos tecnológicos son una herramienta muy importante que facilita la adquisición de materiales y conocimientos de una manera muy rápida, a la vez, que proporciona un medio por el cual también los niños pueden aprender de manera autónoma, tanto de manera individual como grupal.

El uso de las nuevas tecnologías nos ha permitido trabajar de forma colaborativa de una manera más sencilla a través de diferentes herramientas.

Finalment, la majoria dels participants destaquen la seva contribució en l'elaboració de materials propis i en el seu ús a l'aula:

He aprendido a manejar una gran cantidad de aplicaciones tanto online como offline que evidentemente me permitirán plantear actividades diferentes donde mis alumnos podrán disfrutar de múltiples actividades con un gran abanico de posibilidades.

Esta asignatura me ha proporcionado conocer diversidad de herramientas y recursos tecnológicos que anteriormente desconocía, las cuales, en general me han parecido bastante interesantes puesto que nos permiten enfocar el aprendizaje de una manera diferente atendiendo a aspectos como la motivación, la creatividad, la innovación.

Las herramientas tecnológicas me han proporcionado diferentes recursos con los que creo que se podría trabajar en un aula de forma interactiva y divertida. 


\section{Conclusions}

A la vista dels resultats obtinguts, i conscients de la dificultat de valorar percepcions i actituds, observem que els futurs docents realitzen una valoració positiva de les TIC en la seva formació. Aquesta conclusió coincideix amb els resultats d'altres estudis (Domínguez, 2019; Roig-Vila, Mengual-Andrés, Sterrantino i Quinto, 2015).

Els aspectes millor valorats són que faciliten l'intercanvi d'informació a través de diferents mitjans, que resulten fonamentals en la formació permanent del docent, i que les aportacions personals en la xarxa poden afavorir l'aprenentatge d'altres persones. En aquest sentit entenem que la utilització del portafoli digital constitueix un mitjà adequat per a compartir les activitats realitzades i la reflexions sobre el propi aprenentatge.

En canvi, els aspectes que han tingut una valoració més crítica són que la diversitat de serveis en la xarxa dificulta la gestió dels seus continguts i la càrrega de treball addicional que a vegades suposa la seva utilització. Aquesta percepció, derivada de la multiplicitat d'eines existents i la seva ràpida obsolescència, demanda en la formació del professorat el desenvolupament d'estratègies adequades per a la selecció d'aquestes, basades fonamentalment en el seu ús i finalitat.

En l'estudi realitzat també hem pogut comprovar que no existeixen diferències estadísticament significatives en la percepció sobre les TIC en funció del gènere. La percepció sobre el valor de les TIC és lleugerament més positiva per part de les dones que dels homes. Aquests resultats són coincidents amb l'estudi de cas múltiple realitzat per Domínguez (2019) en el context de la formació de Postgrau de futurs docents de Secundària. En els tres casos analitzats la valoració va ser més positiva per part de les dones, no obstant això, igual que en el present estudi les diferències obtingudes no van ser estadísticament significatives. Aquests resultats podrien estar d'acord amb l'ús preferentment acadèmic de la tecnologia per part de les dones, evidenciat en altres investigacions en el context universitari (Imhof, Vollmeyer i Beierlein, 2006).

Finalment, entenem que la utilització del portafoli digital com diari d'aula, i la mateixa realització del qüestionari sobre el valor atorgat a les TIC en la seva formació, han contribuït al fet que els participants reflexionin, fent explícites les seves actituds i creences sobre les tecnologies. 


\section{Referències}

Agra, M.J., Gewerc, A. i Montero, M.L. (2003) «El portafolios como herramienta de análisis en experiencias de formación on-line y presenciales». Enseñanza, 21, p. 101-114.

Alonso-Sanz, M. (2013) «A favor de la Investigación Plural en Educación Artística. Integrando diferentes enfoques metodológicos». Arte, Individuo y Sociedad, 25(1), p. 111-119. doi:10.5209/rev_ARIS.2013.v25.n1.41167

Barragán, R. (2005) «El portafolios, metodología de evaluación y aprendizaje de cara al nuevo Espacio Europeo de Educación Superior». Informática Educativa Comunicaciones, 4(1), p. 121-140.

Blaxter, L., Hugues, C. i Tight, M. (2008) Cómo se investiga. Barcelona, Grao.

Brickner, D. L. (1995) The effects of first and second order barriers to change on the degree and nature of computer usage of mathematics teachers: A case study. (Tesi doctoral). Purdue University, EE. UU.

Domínguez, R. (2019) Entornos personales de aprendizaje en la formación de docentes de Secundaria de la especialidad de Dibujo (Tesi doctoral). Universitat de València, València.

Ertmer, P. A. (1999) «Addressing first- and second-order barriers to change: Strategies for technology integration». Educational Technology Research and Development, 47(4), p. 47-61.

Ertmer, P. A. (2005) «Teacher pedagogical beliefs: The final frontier in our quest for technology integration?». Educational technology research and development, 53(4), p. 25-39.

Gutiérrez, A., Palacios, A. i Torrego, L. (2010) «La formación de los futuros maestros y la integración de las TIC en la educación: anatomía de un desencuentro». Revista de Educación, 353, p. 267-293.

Huerta, R. i Domínguez, R. (2011) «Las TIC en la formación de docentes de la especialidad de dibujo. La propuesta 'aula infinita' para el nuevo master universitario de profesorado en Educación Secundaria». EDUTEC. Revista Electrónica de Tecnología Educativa, 36, p. 1-14. doi:10.21556/edutec.2011.36.399

Imhof, M., Vollmeyer, R. i Beierlein, C. (2006) «Computer use and the gender gap: The issue of Access, use, motivation, and performance». Computers in Human Behaviour, 23, p. 2823-2837. doi:10.1016/j.chb.2006.05.007

Kagan, D. M. (1992) «Implications of research on teacher belief». Educational Psychologist, 27(1), p. 65-90.

Kember, D., Biggs, J. i Leung, D. Y. (2004) «Examining the multidimensionality of approaches to learning through the development of a revised version of the Learning Process Questionnaire». British Journal of Educational Psychology, 74(2), p. 261-279. doi:10.1348/000709904773839879

Lawless, K. i Pellegrino, J. (2007) «Professional Development in Integrating Technology into Teaching and Learning: Knowns, Unknowns, and Ways to Pursue Better Questions ans Answers». Review of Educational Research, 77, p. 575-614. doi:10.3102/0034654307309921

Martínez, A., Dimitriadis, Y., Gómez, E., Rubia, B. i De la Fuente, P. (2003) «Combining qualitative and social network analysis for the study of classroom social interactions». Computers and Education, special issue on Documenting Collaborative Interactions: Issues and Approaches, 41(4), p. 353-368. doi:10.1016/j.compedu.2003.06.001 
Nespor, J. (1987) «The role of beliefs in the practice of teaching». Journal of Curriculum Studies, 19(4), p. 317-328.

Pajares, M.F. (1992) «Teachers' Beliefs and Educational Research: Cleaning up a Messy Construct». Review of Educational Research, 62(3), p. 307-332.

Paraskeva, F., Bouta, H. i Papagianni, A. (2008) «Individual Characteristics and Computer Self-efficacy». Computers \& Education, 50(3), p. 1084-1091. doi:10.1016/j.compedu.2006.10.006

Pelgrum, W. J. (2001) «Obstacles to the integration of ICT in education: Results from a worldwide educational assessment». Computers and Education, 37(2), p. 163-178. doi:10.1016/S0360-1315(01)00045-8

Roig-Vila, R., Mengual-Andrés, S., Sterrantino, C. i Quinto, P. (2015) «Actitudes hacia los recursos tecnológicos en el aula de los futuros docentes». @tic. revista d'innovació educativa, 15, p. 12-19. doi:10.7203/attic.15.7220

Yin, R. K. (2009) Case study research: design and methods. California, Sage. 


\section{Percepciones de los estudiantes sobre el valor de las tecnologías de la información y comunicación en su formación como docentes}

Resumen: Las actitudes y creencias de los docentes en torno a las tecnologías desempeñan un factor clave para la implementación de las mismas en la práctica educativa. Los programas de formación del profesorado en competencia TIC, tanto en la formación inicial como en la permanente, suelen centrarse en la adquisición de una serie de conocimientos y en el desarrollo de ciertas habilidades y destrezas en torno al uso de los recursos tecnológicos, pero no suelen abordar las percepciones o creencias que tienen los docentes sobre su valor en los procesos de enseñanza-aprendizaje. En este artículo se presenta una investigación, realizada en el contexto de la formación inicial de futuros docentes de Primaria, en la que se han generado espacios de autorreflexión sobre el valor y uso de los recursos tecnológicos. A través del cuestionario realizado a final del curso se ha analizado qué aspectos son destacados por los estudiantes sobre el uso de las tecnologías en su formación y en su utilización en el aula.

Palabras clave: Percepciones, creencias, actitudes, tecnologías de la información y comunicación, formación inicial, género.

\section{Perceptions des élèves sur la valeur des technologies de l'information et de la com- munication dans leur formation en tant qu'enseignants}

Résumé: Les attitudes et les croyances des enseignants autour des technologies constituent un facteur clé pour leur mise en œuvre dans la pratique éducative. Les programmes de formation des enseignants en matière de TIC, que ce soit dans la formation initiale ou dans la formation permanente, sont généralement centrés sur l'acquisition d'une série de connaissances et sur le développement de certaines capacités et compétences autour de l'utilisation des ressources technologiques. En revanche, ils n'abordent pas généralement les perceptions ou les croyances des enseignants concernant leur valeur dans les processus d'enseignement-apprentissage. Cet article présente une recherche réalisée dans le contexte de la formation initiale de futurs enseignants de primaire, qui a donné lieu à des espaces d'autoréflexion sur la valeur et l'usage des ressources technologiques. Le questionnaire réalisé à la fin du cours a permis d'analyser les aspects mis en valeur par les étudiants sur l'usage des technologies dans leur formation et sur leur utilisation en classe.

Mots clés: Perceptions, croyances, attitudes, technologies de l'information et de la communication, formation initiale, genre.

\section{Students' perceptions of the value of information and communication technolo- gies in their training as teachers}

Abstract: Teachers' attitudes and beliefs play a key role in the implementation of technologies in educational practice. Teacher training programmes in ICT competence, in both initial and in-service training, usually focus on the acquisition of a body of knowledge and the development of certain skills and abilities around the use of technological resources, but they do not usually address teachers' perceptions or beliefs regarding their value in the teaching-learning processes. This article describes a study carried out in the context of the initial training of future Primary teachers which generate spaces of self-reflection on the value and use of technological resources. The data obtained from a questionnaire administered at the end of the course allow an analysis of the aspects that students highlight with regard to the use of technologies in their training and in their use in the classroom.

Keywords: Perceptions, beliefs, attitudes, information and communication technologies, initial training, gender. 